\title{
Carbon Nanoparticles and Carbonaceous Solids
}

\author{
W. W. Duley \\ Department of Physics and Astronomy, University of Waterloo, \\ 200 University Ave. W. Ontario, Canada \\ email: wwduley@uwaterloo.ca
}

\begin{abstract}
This paper reports on the preparation of hydrogenated amorphous carbon nanoparticles whose spectral characteristics include an absorption band at $217.5 \mathrm{~nm}$ with the profile and characteristics of the interstellar $217.5 \mathrm{~nm}$ feature. Vibrational spectra of these particles also contain the features commonly observed in IR absorption and emission from dust in the diffuse interstellar medium. These materials are produced under "slow" deposition conditions by minimizing the flux of incident carbon atoms and by reducing surface mobility.
\end{abstract}

Keywords. ISM: molecules, ISM: lines and bands, ISM: clouds

Samples of hydrogenated amorphous carbon (HAC) prepared by single atom deposition of carbon at $77 \mathrm{~K}$ results in the formation of nano-particles whose spectroscopic properties include the appearance of a $217.5 \mathrm{~nm}$ absorption band. The characteristics of this band reproduce those of the interstellar feature as catalogued by Fitzpatrick \& Massa (1986). Chemical analysis of this material obtained from XPS, Raman and SERS spectra show that it consists of a network of chains and rings. The ring component is limited to small $s p^{2}$ bonded molecules, including a variety of naphthalene derivatives, which we suggest are the source of the $217.5 \mathrm{~nm}$ absorption band. The spectra contain evidence for acetylenic, aliphatic and olefinic chains containing up to six carbon atoms, as well as hybrid structures involving chains bonded to rings. One of these is diphenylacetylene. Compositions that result in the enhancement of the $217.5 \mathrm{~nm}$ band correspond to an $s p^{2}$ bonded content of $\sim 0.3$ and an $s p^{3}$ bonded content of $\sim 0.7$, implying that deposition under these conditions tends to form carbon chains, rather than rings. These experiments suggest that HAC, in the form of nanoparticles, forms in situ on the surface of cool silicate grains in the DISM. This material has a rich vibrational spectrum that can
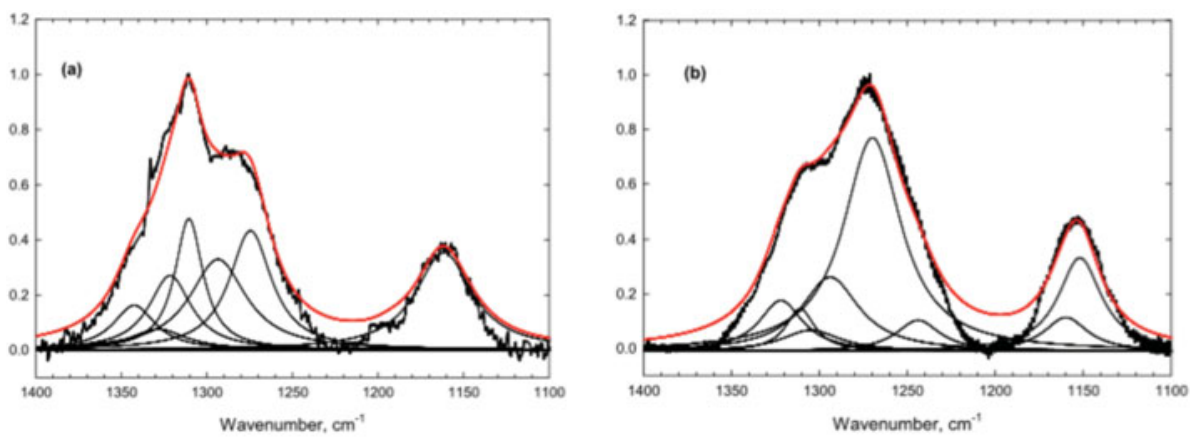

Figure 1. Type A and Type B emission in the middle IR (van Diedenhoven et al. 2004) with fits derived from laboratory spectra. Fit parameters are listed in Table 1. There is a clear distinction between these fits, indicating that the material responsible for this emission is different in the two types of source. 
Table 1. Spectral components appearing in the fits to Type A and Type B emission spectra. These components are seen in spectra of laboratory samples.

\begin{tabular}{cccccc}
$\begin{array}{c}\text { Wavelength } \\
\mu \mathrm{m}\end{array}$ & $\begin{array}{c}\text { Type A } \\
\text { Energy } \\
\mathrm{cm}^{-1}\end{array}$ & $\begin{array}{c}\text { FWHM } \\
\mathrm{cm}^{-1}\end{array}$ & $\begin{array}{c}\text { Wavelength } \\
\mu \mathrm{m}\end{array}$ & $\begin{array}{c}\text { Type B } \\
\mathrm{cm}^{-1}\end{array}$ & $\begin{array}{c}\mathrm{FWHM}^{-1} \\
\mathrm{~cm}^{-1}\end{array}$ \\
\hline 7.446 & 1343 & 15 & 7.446 & 1343 & 15 \\
7.524 & 1329 & 20 & & & \\
7.564 & 1322 & 15 & 7.564 & 1322 & 15 \\
7.628 & 1311 & 10 & 7.628 & 1311 & 10 \\
7.728 & 1294 & 20 & 7.662 & 1305 & 20 \\
7.843 & 1275 & 15 & & 1294 & 20 \\
& & & 7.874 & 1270 & 20 \\
8.382 & 1193 & 10 & 8.039 & 1244 & 15 \\
8.606 & 1162 & 20 & 8.621 & 1160 & 15 \\
& & & 8.681 & 1152 & 15
\end{tabular}

be resolved into a large number of components corresponding to specific modes of the chemical structures contained in these nanoparticles. This spectroscopic data has been used to produce detailed fits to emission in type A and B sources as determined by van Diedenhoven et al. (2004) (Fig. 1) as well as that of the PPN IRAS 01005+7910 (Zhang $\&$ Kwok 2011). We believe that these represent the first accurate fits to astronomical spectra of this kind using purely experimental measurements on carbonaceous materials produced in the laboratory. Further investigation of the properties of these materials is warranted, as they would seem to represent an accurate simulation of interstellar carbon dust.

The model that is indicated from this analysis is that of the in situ formation of hydrogenated amorphous carbon nanoparticles by accretion of individual carbon atoms from the gas phase under DISM conditions. The overall morphology is shown in Duley \& Hu (2012). This accretion occurs on the surface of irregular porous silicate particles and initially leads to the formation of small chains. Above a certain length, these chains become unstable with respect to cyclic structures, particularly to the formation of phenyl. Reactions between radicals also promote ring formation, and as both of these effects occur, the composition evolves into a mixture of ring and chain compounds. Phenyl and naphthyl radicals act as key templates for the formation of a variety of substituted rings and larger structures, but the chemistry continues to be driven by addition reactions involving ethynyl groups being created on the surface by the continued accretion of incident carbon atoms from the ambient gas. This results in HAC particles whose internal composition is enriched in small aromatic rings, and whose outer surface contains a higher concentration of chains and radicals. The chemical energy associated with reactions between radicals and with the recombination of hydrogen atoms trapped within this material is periodically released leading to heating of the particle and to emission of the IR spectral features associated with aromatic hydrocarbons (Duley \& Williams 2011). A comprehensive discussion, together with further experimental data will appear in Duley \& Hu (2012).

This research was supported by the NSERC of Canada.

\section{References}

Duley, W. W. \& Hu, Anming 2012, ApJ 761, 115

Duley, W. W. \& Williams, D. A. 2011, ApJ 737, L44

Fitzpatrick, E. L. \& Massa, D. 1986, ApJ 307, 286

van Diedenhoven, B., Peeters, E., Van Kerckhoven, C., et al. 2004, ApJ 611, 928

Zhang, Y. \& Kwok, S. 2011, ApJ 730, 126 